\title{
Clinical Manifestations of Cuticular Drusen: Current Perspectives
}

\author{
Serena Fragiotta $\mathbb{I D}^{1}$ \\ Pedro Fernández- \\ Avellaneda (iD) $^{2}$ \\ Mark P Breazzano (iD ${ }^{3}$ \\ Gianluca Scuderi (D)' \\ 'NESMOS Department, Ophthalmology \\ Unit, St. Andrea Hospital, University of \\ Rome "La Sapienza", Rome, Italy; \\ ${ }^{2}$ Department of Ophthalmology, Basurto \\ University Hospital, Bilbao, Spain; \\ ${ }^{3}$ Wilmer Eye Institute, Johns Hopkins \\ Hospital, Johns Hopkins University, \\ Baltimore, MD, UA
}

\begin{abstract}
Cuticular drusen are part of the spectrum of age-related macular degeneration (AMD) with particular clinical and multimodal imaging characteristics. This drusen subpopulation shares several high-risk single nucleotide polymorphisms with AMD. Despite this feature, they can manifest at a relatively young age, presenting with a female preponderance. Multimodal imaging is essential for characterizing such lesions, using a combination of color fundus photographs, optical coherence tomography (OCT), fluorescein angiography (FA), and fundus autofluorescence (FAF). The classic starry-sky pattern visible on FA and the typical central hypoautofluorescent lesion with hyperautofluorescent rim on FAF is considered the result of a central retinal pigment epithelium (RPE) erosion from these triangular elevations of the RPE-basal lamina. This finding may also be responsible for the typical choroidal hypertransmission appreciated through OCT. The clinical course of cuticular drusen may be relatively benign at early stages, with small drusen presenting at a young age. However, the presence of clinical phenotypes characterized by diffuse involvement and/ or accompanying large drusen in patients older than 60 years may confer a significant risk for either macular neovascularization or geographic atrophy.
\end{abstract}

Keywords: cuticular drusen, spectral-domain optical coherence tomography, fundus autofluorescence, fluorescein angiography, multimodal imaging

\section{Introduction}

Gass first described cuticular drusen in 1977 as small round yellow subretinal lesions, which appear hyperfluorescent during the arteriovenous phase of fluorescein angiography (FA), characteristically resembling "stars-in-the-sky". ${ }^{1}$ These alterations were initially thought to represent nodular thickening of the basement membrane of the retinal pigment epithelium (RPE), and thus named "basal laminar drusen". ${ }^{2}$ Later, the histopathological correlation demonstrated that basal laminar drusen share with conventional drusen the localization between RPE-basal lamina (BL) and Bruch's membrane (BrM). ${ }^{3}$ Therefore, the term "cuticular" drusen was preferred to connotate a similar ultrastructure to conventional drusen. ${ }^{2,4,5}$ Although sharing a similar content with hard drusen, cuticular drusen are numerous and tend to coalescence, resulting in a peculiar diffuse distribution. ${ }^{6}$

Major advances in multimodal imaging have permitted an accurate characterization of the cuticular drusen, identifying imaging criteria that allow a distinction with other drusen subtypes. In particular, the size and spatial density of cuticular drusen can be quantified with greater precision through imaging techniques compared to clinical examination. ${ }^{6}$ The multimodal recognition has elucidated the natural course, prognosis, and macular complications associated with cuticular
Correspondence: Serena Fragiotta NESMOS Department, Ophthalmology Unit, St. Andrea Hospital, University of Rome "La Sapienza", Via di Grottarossa 1035-1039, Rome, 00189, Italy

Tel +393293276433

Fax +390633776628

Email s.fragiotta@hotmail.it 
drusen. ${ }^{7,8}$ In fact, similar to soft drusen, cuticular drusen exhibited a dynamic lifecycle with growth, coalescence and resorption leading to RPE abnormalities and subsequent development of macular complications. ${ }^{6,9-11}$

The present review aims to provide a comprehensive overview of the existing literature on the wide spectrum of clinical manifestations associated with cuticular drusen in age-related macular degeneration (AMD), offering insights into clinical characteristics, multimodal characterization, phenotypes, natural course, and prognosis of cuticular drusen.

\section{Clinical Aspects of Cuticular Drusen Demographic Characteristics and Genetic Factors}

Cuticular drusen are present in the spectrum of AMD in most cases, sharing clinical features and complications. However, the relatively younger age and female preponderance are demographic features that characterize this population, divergent from typical AMD. ${ }^{6,7,12}$ Overall, the female prevalence varied from 60 to $92.1 \%$ in the cuticular population, particularly when accompanied by macular complications. ${ }^{6,7,13}$ Patients with a confirmed diagnosis of cuticular drusen were five times more likely to be female. ${ }^{14}$ The female preponderance in the cuticular subgroup seems to be peculiar considering the absence of gender difference both in early and late AMD found in large cohort studies. ${ }^{15,16}$

Genetic risk factors have been poorly investigated, but a strong association with single nucleotide polymorphism on the CFH gene (p.Tyr402His) has been reported in patients with the cuticular phenotype of drusen. ${ }^{17}$ However, the frequency of the $C F H \mathrm{Y} 402 \mathrm{H}$ alleles in eyes presenting with a vitelliform detachment associated with cuticular drusen was similar to the general population, suggesting a distinct clinical entity. ${ }^{18}$

Genetic variants of the $\mathrm{CFH}$ gene were identified in $8.8 \%$ of patients with cuticular drusen subtype of AMD, and patients carrying rare $\mathrm{CFH}$ variants presented an earlier age at onset. Moreover, a rare missense variant $\mathrm{p}$. Arg1210Cys was putatively identified as specific for the cuticular phenotype. ${ }^{19}$ Mutations in the fibulin-5 gene (FBLN5), coding for an extracellular matrix protein at the level of Bruch's membrane, have been also associated with cuticular drusen. ${ }^{20,21}$

The estimated frequency of histidine allele at the Tyr402His locus was $70 \%$ in AMD patients with cuticular drusen, which was higher than the conventional AMD cohort (55\%), corroborating a primary role of the complement cascade in the pathogenesis of this drusen subtype. ${ }^{13}$

Cuticular drusen population shared with AMD several high-risk single nucleotide polymorphisms, including rs1410996 (CFH), rs10490924 (ARMS2), rs4151667 (CFB), rs9332739 (C2), rs2230199 (C3), rs7412 (APOE E2), and rs429358 (APOE E4). This observation suggests a possible common genetic background between these AMD subtypes. ${ }^{22}$

\section{Membranoproliferative Glomerulonephritis (MPGN)}

Beyond the spectrum of AMD, cuticular drusen have been described in association with membranoproliferative glomerulonephritis (MPGN) type II. This rare form of chronic glomerulonephritis usually affects children and its clinical course is characterized by rare remissions and progression to end-stage renal disease. ${ }^{23}$

Characteristically, the ultrastructural findings include the accumulation of intramembranous osmiophilic dense deposits at the level of lamina densa of the glomerular basement membrane, and similar deposits, can accumulate at the level of the choriocapillaris-Bruch's membraneretinal pigment epithelium in the affected individuals. ${ }^{23,24}$

Drusen-like deposits resembling cuticular drusen were observed in all the subjects with long-standing MPGN disease ( $>16$ months). The deposits are barely observed on clinical examination and well-visualized on the arteriovenous phase of fluorescein angiography. Along with MPGN progression over time ( $>15$ years), retinal findings can exhibit further complications including atrophy of the retina and RPE as well as macular neovascularization. ${ }^{25}$ Similar findings were also associated with $\mathrm{C} 3$ glomerulonephritis, corroborating abnormalities in the the complement pathway for the pathogenesis of drusen in the context of complement-mediated glomerular diseases. ${ }^{26}$

\section{Clinical Appearance and Diagnostic Criteria}

Cuticular drusen appear clinically as a myriad of roundish yellow lesions, clustering in the macular region and/or mid-peripheral retina. They typically measure between 50 and $75 \mu \mathrm{m}$ in diameter. ${ }^{1,8,12}$

Nevertheless, the clinical appearance alone is not distinctive enough to differentiate with other drusen subtypes. Particularly when located preferentially in the peripheral retina, they can be mistaken with hard drusen, or if they grow and coalesce can be misinterpreted as soft drusen. ${ }^{8,27}$ 
Based on topographical distribution, cuticular drusen have been distinguished into three phenotypes: ${ }^{7}$

Phenotype 1: clusters of numerous lesions concentrated in the macular region and occasionally extending beyond vascular arcades and peripapillary region.

Phenotype 2: lesions scattered around the posterior pole, extending into the peripheral retina and nasally past the optic nerve.

Phenotype 3: mixed type associated with large drusen $(>200 \mu \mathrm{m})$, likely representing hyalinized or large colloid drusen.

Diagnostic criteria were proposed based on multimodal imaging observations that assist in accurate differentiation from other similar drusen phenotypes. ${ }^{6,7}$ A diagnosis can be made using at least three of four imaging methods, including color fundus photographs, spectral-domain optical coherence tomography (SD-OCT), FA, and fundus autofluorescence (FAF). ${ }^{6}$

\section{Multimodal Characterization of Cuticular Drusen}

Multimodal imaging characteristics probably reflect the internal composition of these drusen subtypes. Features of different subtypes distinguished by multimodal imaging are summarized in Table 1.

\section{Fundus Autofluorescence}

Clinical examination alone is not sufficient to reveal cuticular drusen, especially in the early stages with subtle lesions exhibiting poor contrast. FAF can instead detect these drusen prior to eruption through the RPE. The classic FAF pattern is characterized by a central hypoautofluorescence with a hyperautofluorescent rim (Figure 1). ${ }^{6,28}$

The central RPE attenuation in these drusen is mainly localized at the apex with normal thickening at the drusen edge. Thus, less pigment blocks the excitation wavelength, and explains this produced typical FAF pattern. ${ }^{8}$ A limitation when interpreting FAF is media opacity, for example, lenticular nuclear sclerosis, which may obstruct visualization of FAF details. Consequently, cuticular drusen have been correctly identified as present in $62 \%$ of cases and not present in $100 \%$ of cases. ${ }^{14}$ In this regard, the use of a long-wavelength excitation $(530-580 \mathrm{~nm})$ can potentially minimize autofluorescence from the lens and increase the ability to detect heterogeneity in foveal autofluorescence due to RP/E alterations. ${ }^{29}$

Confocal scanning laser ophthalmoscopy (cSLO) with real-time tracking improves the visualization of drusencontaining regions allowing an integrated approach with OCT b-scan. The main advantages in using a cSLO FAF $(488 \mathrm{~nm})$ reside in the diffuse availability and the simultaneous integration with other imaging techniques as color fundus photography, FA, and SD-OCT. ${ }^{29,30}$

\section{Fluorescein Angiography and Indocyanine Green Angiography}

The typical appearance of cuticular drusen is represented by the "starry-sky" pattern characterized by

Table I Multimodal Imaging Characteristics of the Different Drusen Subtypes

\begin{tabular}{|c|c|c|c|c|}
\hline & Hard Drusen & Soft Drusen & Cuticular Drusen & Subretinal Drusenoid Deposits \\
\hline CFP & $\begin{array}{l}\text { Small }(<63 \mu \mathrm{m}) \text { discrete } \\
\text { yellowish deposits with } \\
\text { defined margins. }\end{array}$ & $\begin{array}{l}\text { Larger }(125 \mu \mathrm{m}) \\
\text { yellow-white elevations } \\
\text { and indistinct edges. }\end{array}$ & $\begin{array}{l}\text { Numerous small }(25- \\
75 \mu \mathrm{m}) \text { dot-like } \\
\text { yellow deposits }\end{array}$ & $\begin{array}{l}\text { White dots or reticular appearance mainly located in } \\
\text { the perifovea }\end{array}$ \\
\hline FAF & $\begin{array}{l}\text { Focal points of reduced } \\
\text { AF }\end{array}$ & Slightly hyper-AF & $\begin{array}{l}\text { Hypo-AF dots with } \\
\text { hyper-AF halo }\end{array}$ & Hypo-AF, hyper-AF in some cases \\
\hline OCT & Focal RPE-BL elevations & $\begin{array}{l}\text { Mound RPE-BL } \\
\text { elevations }\end{array}$ & & $\begin{array}{l}\text { Near infrared reflectance revealed dot or ribbon } \\
\text { pattern; OCT b scan: hyperreflective deposits between } \\
\text { the RPE and the ellipsoid zone; }\end{array}$ \\
\hline FA & Mild hyperfluorescence & $\begin{array}{l}\text { Variable- usually late } \\
\text { hyperfluorescence }\end{array}$ & $\begin{array}{l}\text { Hyperfluorescent } \\
\text { dots with "stars-in- } \\
\text { the-sky" appereance }\end{array}$ & Hypofluorescent or no changes \\
\hline ICGA & Hyperfluorescent & Hypofluorescent & $\begin{array}{l}\text { Early } \\
\text { hyperfluorescence }\end{array}$ & Hypofluorescence in mid-to-late phases \\
\hline
\end{tabular}

Abbreviations: CFP, color fundus photograph; FAF, fundus autofluorescence; OCT, optical coherence tomography angiography; FA, fluorescein angiography; ICGA, indocyanine green angiography; RPE, retinal pigment epithelium; BL, basal lamina. 


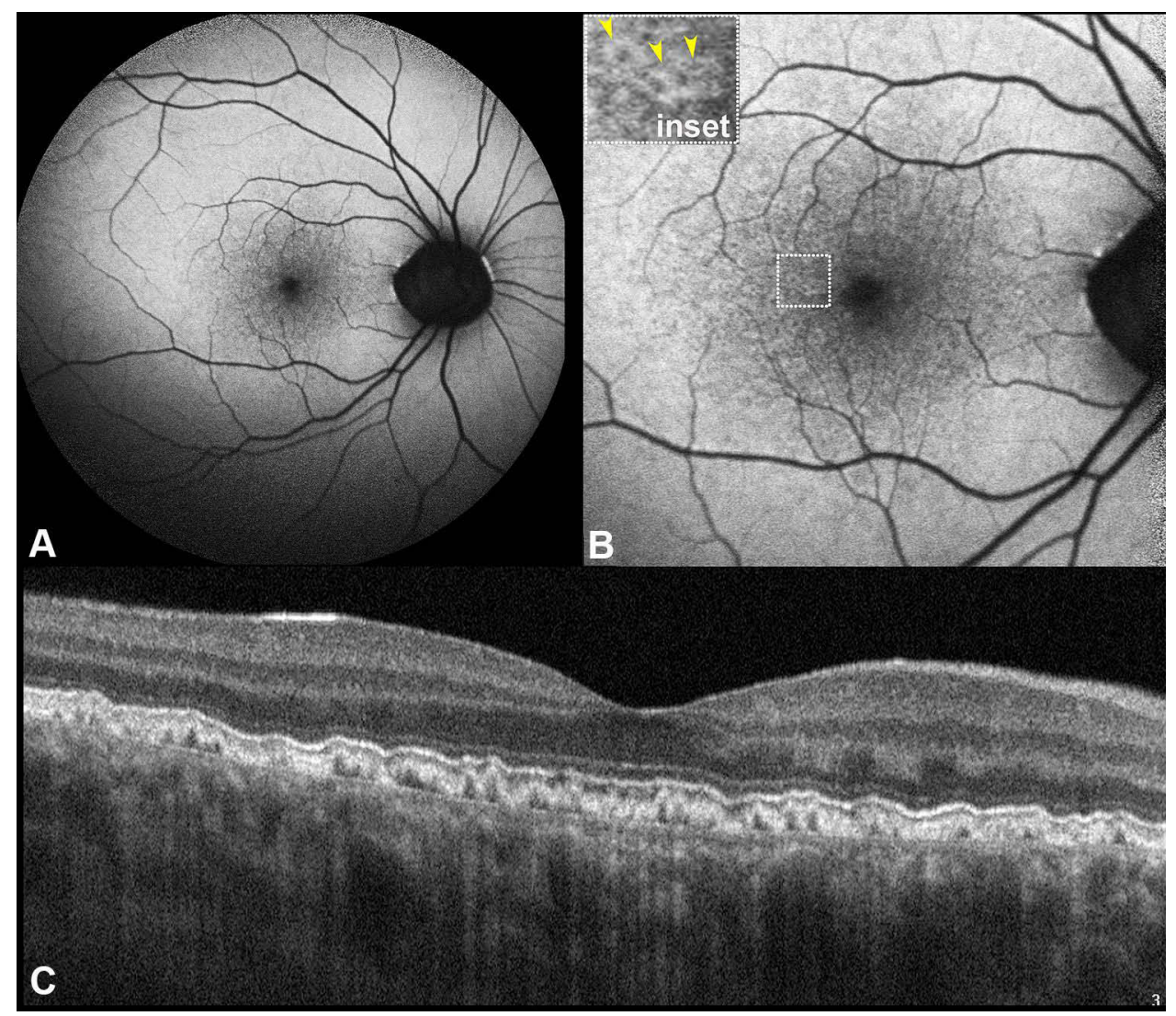

Figure I Short wavelength fundus autofluorescence (FAF). (A) A 55-degree FAF acquired using a Spectralis (Heidelberg Engineering, Heidelberg, Germany) device that uses a $488 \mathrm{~nm}$ illumination wavelength and emission detection between 500 and $700 \mathrm{~nm}$; FAF imaging demonstrated pinpoint hypoautofluorescent alterations confined within the posterior pole. (B) A 35-degree FAF magnification shows more easily the typical pattern of cuticular drusen constituted by central hypofluorescence surrounded by a hyperautofluorescent halo (yellow arrowheads, inset). (C) Subfoveal optical coherence tomography B-scan demonstrating multiple retinal pigment epithelium-basal lamina elevations with a classical saw tooth configuration.

several roundish hyperfluorescent lesions during the arteriovenous phase. ${ }^{1}$ These lesions remained hyperfluorescent for the entire duration of the FA, slowly fading through the very late phases (Figure 2). Meanwhile, indocyanine green angiography (ICGA) showed early hyperfluorescent lesions surrounded by faint hypofluorescent halos becoming intensely hyperfluorescent in the late ICGA phase. ${ }^{30}$ Of note, in about $50 \%$ of cases, the ICGA could not demonstrate drusen appearance despite detection with other imaging methods. ${ }^{6}$

The "starry-sky" pattern can account for the central RPE erosion with multiple associated window defects. In the early stages, when the RPE is intact, the "starry-sky" appearance may not be visible by FA. ${ }^{28}$ However, dye-based angiography techniques (FA and/ or ICGA) are usually performed if deemed clinically necessary to exclude neovascularization ${ }^{7}$ and thus not to provide a primary source of drusen characterization.

\section{Optical Coherence Tomography (OCT)}

\section{Signatures}

On OCT B-scans, cuticular drusen demonstrate triangular elevations of the RPE-BL with bases lying on Bruch's membrane and apices toward the retina, conferring a characteristic "saw-tooth" pattern. In addition, the thinnest RPE apex may account for the typical hypertransmission in correspondence of the drusen center and light attenuation at the edges (Figure 3). 8,31

However, the saw-tooth pattern should not be considered pathognomonic, as it is not discernible in all cases. For this reason, Balaratnasingam et $\mathrm{al}^{6}$ identified three distinct patterns seen on OCT B-scans as categorized below: 


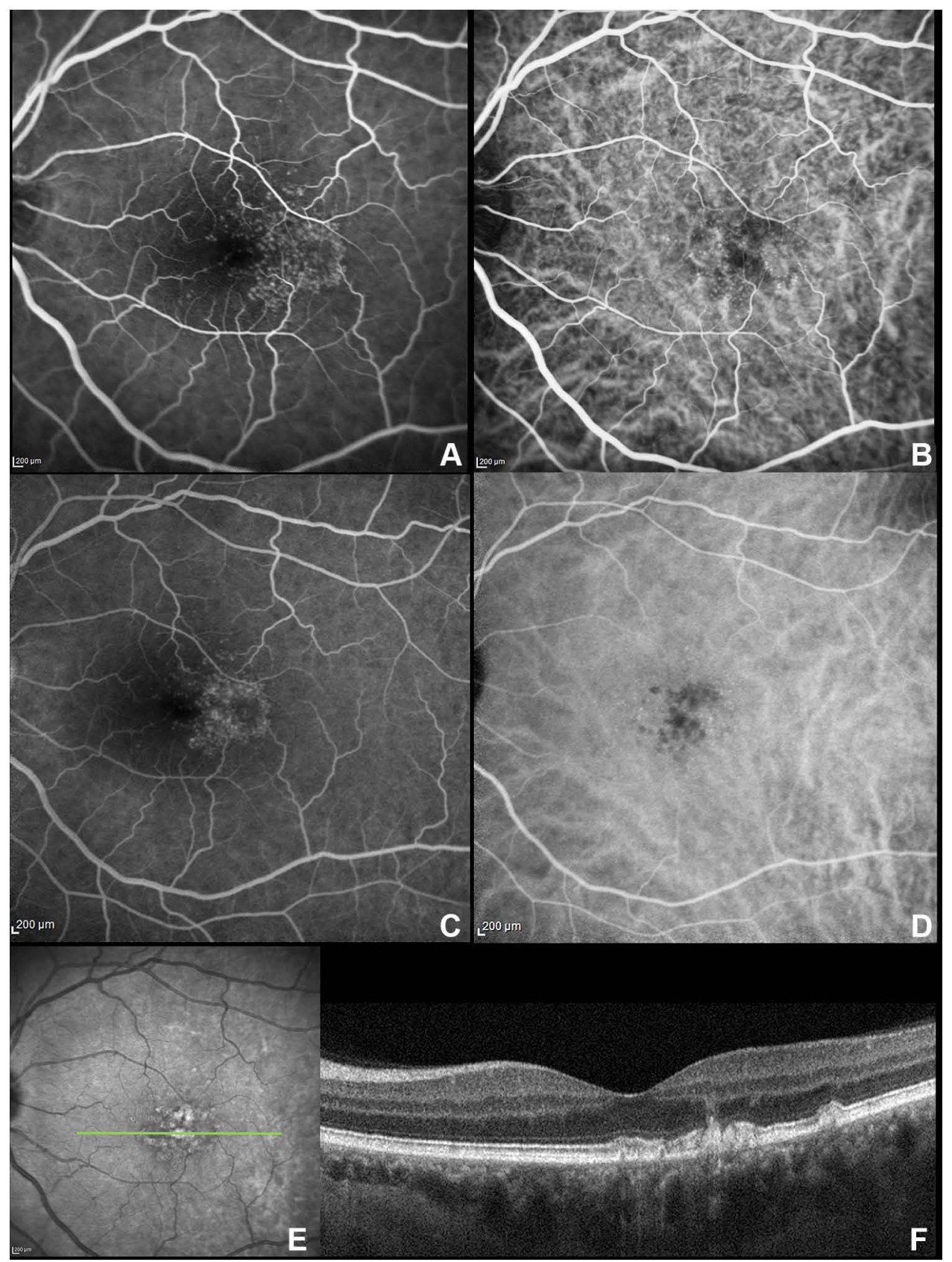

Figure 2 Fluorescein angiography (FA) and indocyanine green angiography (ICGA). Arteriovenous phase (0:43 seconds) showing the typical "stars-in-the-sky" appearance at both FA (A) and ICGA (B); The hyperfluorescent lesions tend to progressively fade, but persist through late phase angiograms (5:02 min) on both FA (C) and ICGA (D). (E) Infrared reflectance with scan reference (green line) of the subfoveal optical coherence tomography B-scan (F) showing multiple elevations of the retinal pigment epitheliumbasal lamina with variable content.

1. Type 1 pattern (33\%) - shallow RPE-basal lamina (BL) elevations without a clear discernible drusen content.

2. Type 2 pattern (49\%) - the classical saw-tooth configuration with a hyporeflective internal content.

3. Type 3 pattern (18\%)-broad, mound-shaped RPE-BL elevations with hyporeflective internal content.

\section{Cuticular Drusen Lifecycle}

\section{Cuticular Drusen and Other Drusen}

\section{Subtype}

Large drusen $(>200 \mu \mathrm{m})$, embodying the type 3 pattern according to Sakurada et $\mathrm{al}^{7}$ classification, variably accompanied cuticular drusen in $25-59.3 \%$ of cases (Figure 4). ${ }^{32,33}$ The presence of large drusen was often 


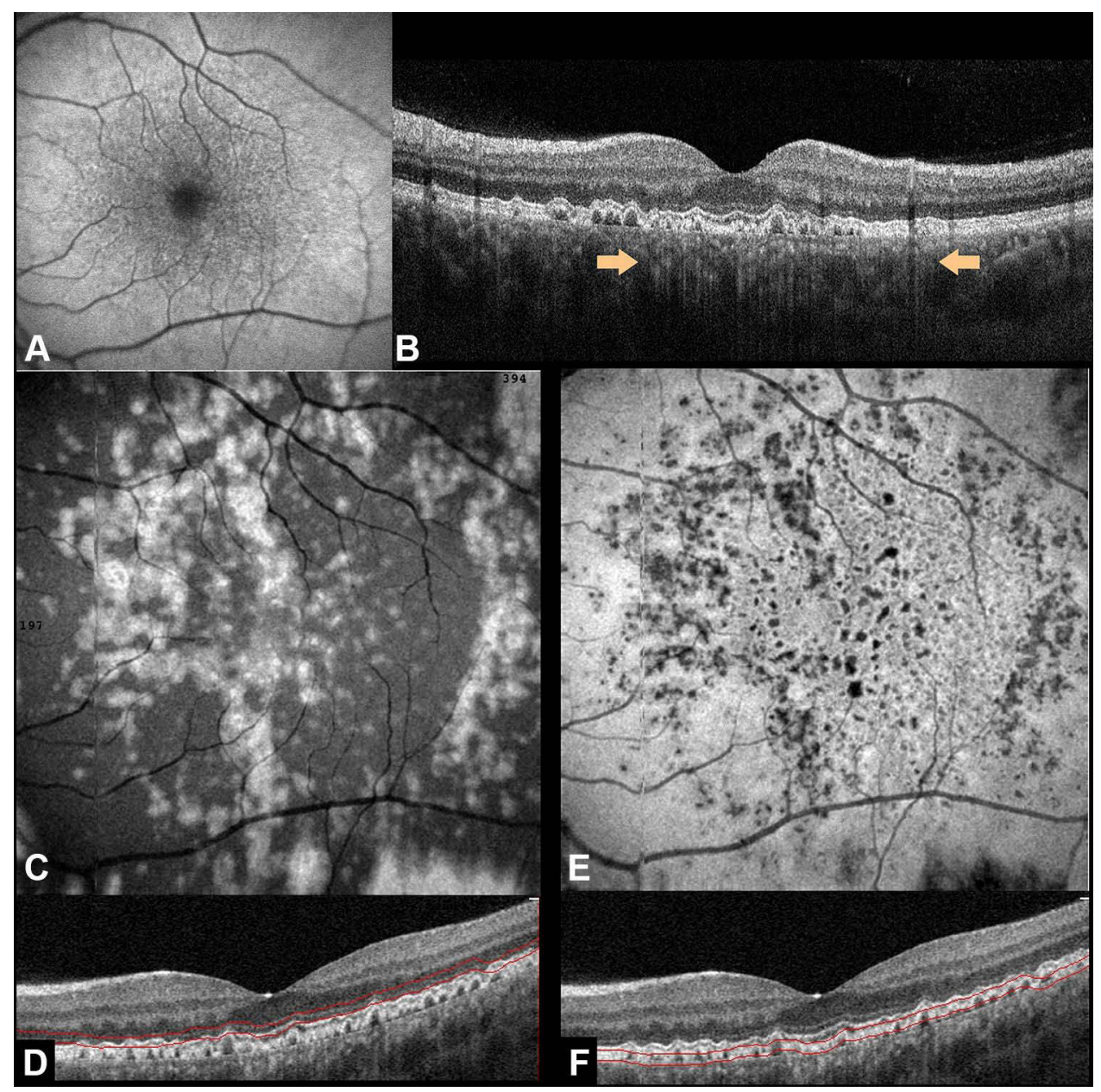

Figure 3 Cuticular drusen recognition using multimodal imaging. (A) Fundus autofluorescence demonstrates multifocal central hypoautofluorescence with hyperautofluorescent border. (B) Spectral-domain optical coherence tomography (OCT) B-scan exhibiting the classical saw-tooth configuration with a hyporeflective internal content and a characteristic bar-code signature (peach arrows) in the choroid. (C) Structural en face obtained through a customized segmentation (D) passing above the plane of drusen $(-95,-55 \mu \mathrm{m}$ offsets) demonstrates their distribution. A structural en face customized view $(-34,6 \mu \mathrm{m}$ offsets) passing through the drusen (E and $\mathbf{F})$ highlights the hyporeflective cores of the cuticular drusen.

accompanied by pigmentary abnormalities in patients aged older than 60 years. $^{6}$ It has been proposed that large colloid drusen may constitute a variant of cuticular drusen, but further studies are necessary to confirm this hypothesis. $^{\text {? }}$

Large colloid drusen, also known as multifocal serous retinal pigment epithelium detachment by Gass, presented a similar clinical appearance of soft drusen and serous pigment epithelial detachment. ${ }^{34,35}$ Despite this, the histopathological findings demonstrated the absence of basal linear and basal laminar deposits, calcification of Bruch membrane, and thickening of the choriocapillaris walls. However, a small focus of choroidal neovascularization with evidence of exudation has been demonstrated on histopathological evaluation. ${ }^{34}$
Although rare, cuticular drusen may present in association with subretinal drusenoid deposits (SDD, also known as reticular pseudodrusen). ${ }^{36}$

Another interesting finding in the cuticular drusen population is the peripapillary involvement that is quite common, reported between 63.3 and $75.3 \%$ of eyes. ${ }^{6,32}$

Upon functional analysis, cuticular drusen present with similar retinal sensitivity values to soft and reticular pseudodrusen/SDD on both mesopic and dark-adapted red conditions, while the dark-adapted cyan condition demonstrated a significantly lower sensitivity for reticular pseudodrusen/SDD with respect to cuticular drusen. A dark-adapted cyan mean defect exceeding the darkadapted red defect suggested a predominant rod dysfunction in reticular pseudodrusen. At the same time, the 


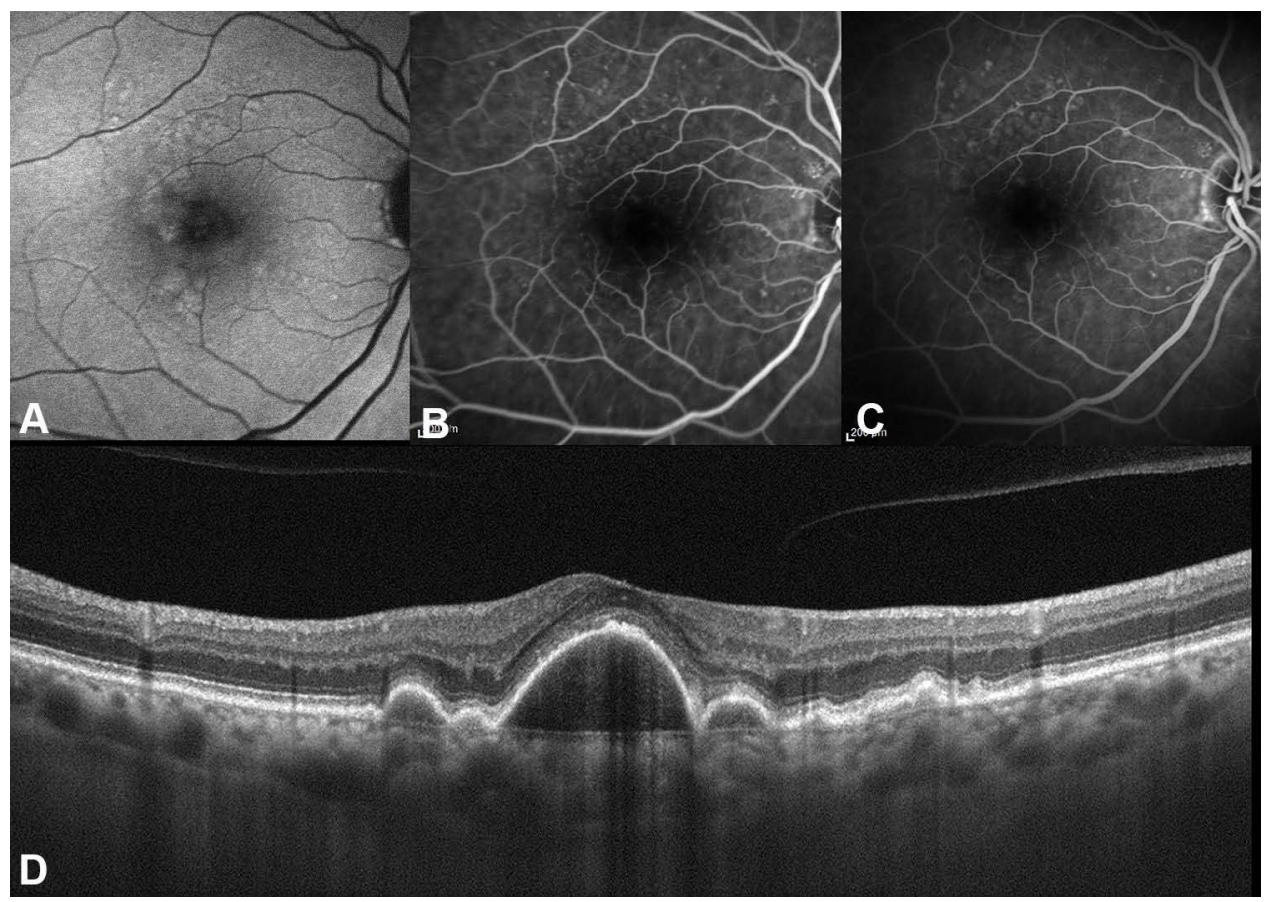

Figure 4 Cuticular drusen associated with large drusen. (A) Fundus autofluorescence shows the presence of large drusen appearing as faint hyperautofluorescent lesions. These large drusen are barely visible on fluorescein angiography during early phases (B) with minimal increase of hyperfluorescence during late phases (C). On spectraldomain optical coherence tomography B-scan through the foveal center, large drusen tend to coalesce into a drusenoid pigment epithelial detachment (D).

positive cyan-red difference for cuticular drusen indicated an isolated foveal cone dysfunction. ${ }^{37}$

\section{Vitelliform Detachment Associated with Cuticular Drusen}

One of the first clinical associations described for cuticular drusen was the vitelliform macular lesion. ${ }^{2}$ Patients with the combination of vitelliform macular detachment and cuticular drusen were younger and presented with worse visual acuity when compared with adult-onset vitelliform macular dystrophy. ${ }^{38}$ The prevalence of acquired vitelliform lesions in patients with cuticular drusen varied by ethnic group from $1.2 \%$ in the Asian population to $24.2 \%$ in Caucasians. ${ }^{6,32}$

These lesions are usually bilateral, characterized by a myriad of drusen producing the typical "starry-sky" appearance, accompanied by a late hyperfluorescence, likely representing the late staining of the vitelliform material (Figure 5). ${ }^{39}$ The vitelliform macular detachment on OCT appears as hyperreflective subretinal material with an overlying hyporeflective space, thus conferring the pseudohypopyon appearance in some cases. ${ }^{30,38}$

The fate of vitelliform detachment associated with cuticular drusen includes collapse with resolution of the vitelliform material and accompaniment of progressive RPE failure and atrophy with drusen disappearance. Moreover, the lesions may lead to development of macular neovascularization (MNV) among 30\% of cases, which can be ill-defined through fluorescein angiography in half of these due to diffuse accumulation of fluorescein in late phases. ${ }^{38,40}$ Long-term vitelliform detachment can progressively affect photoreceptors and RPE atrophy accompanied by the resorption of vitelliform material and progressive choroidal thinning. However, the formation of vitelliform material may modulate the choroidal vasculature thickness with hyperpermeability in the early vitelliform stages, before atrophic changes have developed. ${ }^{41-43}$

\section{Late-stage Macular Complications} Macular Neovascularization (MNV)

MNV has been detected in $12.5 \%$ of cases, among these $76.7 \%$ included type 1 neovascularization, while the remaining cases were mixed type 1 and 2 lesions. $^{6}$ Interestingly, phenotypes 2 and 3 presented a higher risk for the development of MNV, which has been hypothesized to be related to the greater drusen volume than phenotype $1 .^{7}$ Of note, MNV is more frequent in patients older than 60 years, although it can occur earlier in life. ${ }^{6,44}$ 


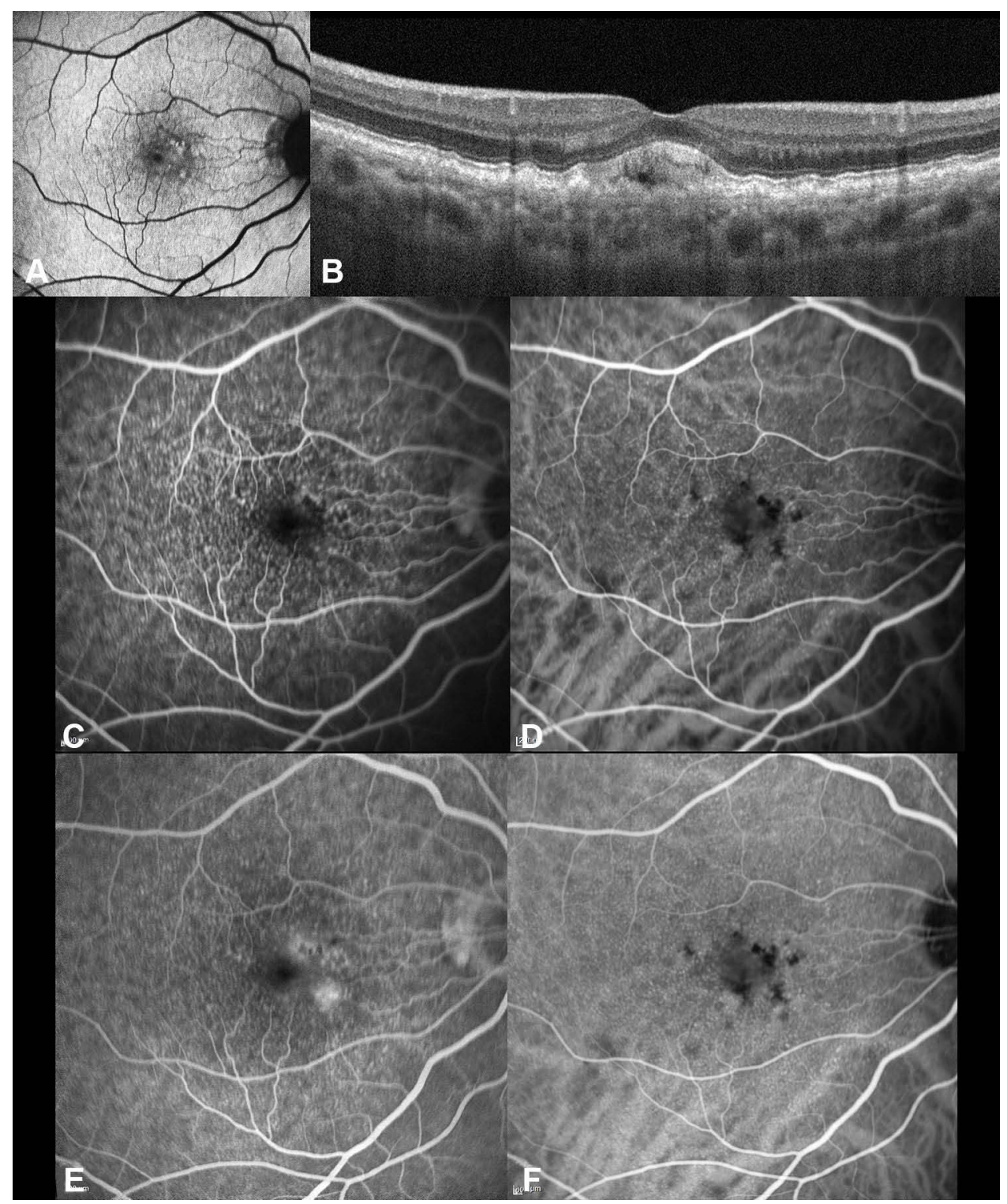

Figure 5 Cuticular drusen associated with vitelliform detachment. (A) Fundus autofluorescence demonstrating hyperautofluorescent signal in correspondence of vitelliform material.; (B) Subfoveal optical coherence tomography B-scan shows discrete retinal pigment epithelium-basal lamina (BL) elevations compatible with cuticular drusen and a subretinal accumulation of hyperreflective material consistent with a vitelliform detachment. (C) Fluorescein angiography (FA) at 0:50 seconds demonstrates a starry-sky appearance; this aspect is also detectable through indocyanine green angiography (ICGA) visible at (D) The starry-sky appearance is maintained in late angiograms (6:42 min) of FA (E) and ICGA (F). Additionally, late staining of vitelliform material can be appreciated with FA, while a central hypofluorescence is visible with ICGA.

Other types of neovascularization have rarely been observed with only few reports so far. ${ }^{7,45,46}$ A case from a 65 -year-old Japanese woman presenting with the typical "stars-in-the-sky" exhibited a type 3 neovascularization lesion. ${ }^{45}$ Another case of a 61-year-old woman of European descent demonstrated bilateral aneurysmal type 1 neovascularization (polypoidal choroidal vasculopathy) in an extrafoveal location along vascular arcade in one eye and the nasal mid-periphery in the fellow eye. Of note, the neovascular lesions co-localized with the drusen distribution that were scattered around the posterior pole, occupying the periphery and retina nasal to the optic nerve, and thus configured cuticular drusen phenotype $2^{7,46}$ Type 2 neovascularization associated with cuticular drusen was diagnosed in only one case in a type $2 \mathrm{MNV}$ cohort with an overall prevalence of $4.3 \%{ }^{47}$

\section{Geographic Atrophy}

Cuticular drusen exhibit dynamic change with growth and coalescence and regression accompanied by RPE alterations like conventional drusen. Drusen loads, represented by the high number of lesions that may aggregate in some cases, 
may contribute to the development of RPE pigmentary changes and GA. ${ }^{6,48,49}$ However, some areas in the fundus can also demonstrate a spontaneous resolution of the drusen, resulting in small defects or "imprint" of the RPE. ${ }^{7}$

Geographic atrophy (GA) occurred in $18.5-25 \%$ of cases, more frequently in patients $>60$ years old. ${ }^{6,7,32}$ However, $9.2 \%$ developed GA among patients 60 years of age or younger. ${ }^{6}$ Like MNV, phenotypes 2 and 3 presented a greater five-year estimated incidence of GA of $38.7 \%$ and $43.6 \%$, respectively, compared to phenotype $1(12.9 \%){ }^{7}$

\section{Conclusions}

Cuticular drusen represent a phenotype of clinical relevance in the spectrum of AMD. These lesions can occur at a younger age than conventional drusen, demonstrating a female preponderance. Therefore, multimodal characterization is essential for accurate drusen phenotyping. In fact, cuticular drusen may coexist with large drusen $(>200 \mu \mathrm{m})$ or large colloidal drusen, resulting from coalescence or growth of typical cuticular drusen, but this needs to be proven.

The presence of cuticular drusen at early stages may be deemed a relatively benign phenomenon, but some additional factors should be considered. In young patients, the co-occurrence of vitelliform lesions can complicate the prognosis for the development of macular complications at a relatively early age. In addition, the presence of phenotypes 2 and 3 in patients older than 60 years may confer a significant risk for either MNV or GA.

The increasing recognition of cuticular drusen made possible by multimodal imaging is essential for identifying patients at risk of developing macular complications at a relatively young age. Macular complications are connected to diffuse RPE disturbances, influenced by drusen dynamic changes and drusen load, varying from vitelliform detachment to classic late-stage complications.

\section{Disclosure}

The authors report no conflicts of interest in this work.

\section{References}

1. Gass JDM. Stereoscopic Atlas of Macular Diseases. Diagnosis and Treatment. Mosby CV, 2nd ed. St.Louis; 1977

2. Gass JD, Jallow S, Davis B. Adult vitelliform macular detachment occurring in patients with basal laminar drusen. Am J Ophthalmol. 1985;99(4):445-459. doi:10.1016/0002-9394(85)90012-1

3. Russell SR, Mullins RF, Schneider BL, Hageman GS. Location, substructure, and composition of basal laminar drusen compared with drusen associated with aging and age-related macular degeneration. Am J Ophthalmol. 2000;129(2):205-214. doi:10.1016/S0002-9394(99) 00345-1
4. Sarks SH, Arnold JJ, Killingsworth MC, Sarks JP. Early drusen formation in the normal and aging eye and their relation to age related maculopathy: a clinicopathological study. $\mathrm{Br} J$ Ophthalmol. 1999;83(3):358-368. doi:10.1136/bjo.83.3.358

5. Farkas TG, Krill AE, Sylvester VM, Archer D. Familial and secondary drusen: histologic and functional correlations. Trans Am Acad Ophthalmol Otolaryngol. 1971;75(2):333-343.

6. Balaratnasingam C, Cherepanoff S, Dolz-Marco R, et al. Cuticular drusen: clinical phenotypes and natural history defined using multimodal imaging. Ophthalmology. 2018;125(1):100-118. doi:10.1016/j. ophtha.2017.08.033

7. Sakurada Y, Parikh R, Gal-Or O, et al. Cuticular drusen: risk of geographic atrophy and macular neovascularization. Retina. 2018:40(2):257-265.

8. Spaide RF, Curcio CA. Drusen characterization with multimodal imaging. Retina. 2010;30(9):1441-1454. doi:10.1097/IAE.0b013e 3181ee5ce8

9. Ouyang Y, Heussen FM, Hariri A, Keane PA, Sadda SR. Optical coherence tomography-based observation of the natural history of drusenoid lesion in eyes with dry age-related macular degeneration. Ophthalmology. 2013;120(12):2656-2665. doi:10.1016/j.ophtha.20 13.05.029

10. Waldstein SM, Vogl WD, Bogunovic H, Sadeghipour A, Riedl S, Schmidt-Erfurth U. Characterization of drusen and hyperreflective foci as biomarkers for disease progression in age-related macular degeneration using artificial intelligence in optical coherence tomography. JAMA Ophthalmol. 2020;138(7):740-747. doi:10.1001/ jamaophthalmol.2020.1376

11. Fragiotta S, Rossi T, Cutini A, Grenga PL, Vingolo EM. Predictive factors for development of neovascular age-related macular degeneration: a spectral-domain optical coherence tomography study. Retina. 2018;38(2):245-252. doi:10.1097/IAE.0000000000001540

12. Boon CJ, van de Ven JP, Hoyng CB, den Hollander AI, Klevering BJ. Cuticular drusen: stars in the sky. Prog Retin Eye Res. 2013;37:90-113. doi:10.1016/j.preteyeres.2013.08.003

13. Grassi MA, Folk JC, Scheetz TE, Taylor CM, Sheffield VC, Stone EM. Complement factor $\mathrm{H}$ polymorphism p.Tyr402His and cuticular drusen. Arch Ophthalmol. 2007;125(1):93-97. doi:10.10 01/archopht.125.1.93

14. Hoeg TB, Moldow B, Klein R, et al. An evaluation of fundus photography and fundus autofluorescence in the diagnosis of cuticular drusen. Br J Ophthalmol. 2016;100(3):378-382. doi:10.1136/ bjophthalmol-2015-307197

15. Wong WL, Su X, Li X, et al. Global prevalence of age-related macular degeneration and disease burden projection for 2020 and 2040: a systematic review and meta-analysis. Lancet Glob Health. 2014;2(2):e106-116. doi:10.1016/S2214-109X(13)70145-1

16. Klein R, Klein BEK, Linton KLP. Prevalence of age-related maculopathy: the Beaver Dam eye study. Ophthalmology. 2020;127(4): S122-S132. doi:10.1016/j.ophtha.2020.01.033

17. van de Ven JP, Boon CJ, Fauser S, et al. Clinical evaluation of 3 families with basal laminar drusen caused by novel mutations in the complement factor H gene. Arch Ophthalmol. 2012;130 (8):1038-1047. doi:10.1001/archophthalmol.2012.265

18. Barbazetto IA, Yannuzzi NA, Klais CM, et al. Pseudo-vitelliform macular detachment and cuticular drusen: exclusion of 6 candidate genes. Ophthalmic Genet. 2007;28(4):192-197. doi:10.1080/ 13816810701538596

19. Duvvari MR, Saksens NT, van de Ven JP, et al. Analysis of rare variants in the $\mathrm{CFH}$ gene in patients with the cuticular drusen subtype of age-related macular degeneration. Mol Vis. 2015;21:285-292.

20. Kucukevcilioglu M, Patel CB, Stone EM, Russell SR. Clinically detectable drusen domains in fibulin-5-associated age-related macular degeneration (AMD): drusen subdomains in fibulin-5 AMD. Int Ophthalmol. 2016;36(4):569-575. doi:10.1007/s10792-0150164-5 
21. Stone EM, Braun TA, Russell SR, et al. Missense variations in the fibulin 5 gene and age-related macular degeneration. $N$ Engl J Med. 2004;351(4):346-353. doi:10.1056/NEJMoa040833

22. van de Ven JP, Smailhodzic D, Boon CJ, et al. Association analysis of genetic and environmental risk factors in the cuticular drusen subtype of age-related macular degeneration. Mol Vis. 2012;18:2271-2278.

23. Appel GB, Cook HT, Hageman G, et al. Membranoproliferative glomerulonephritis type II (dense deposit disease): an update. $\mathrm{J} \mathrm{Am}$ Soc Nephrol. 2005;16(5):1392-1403. doi:10.1681/ASN.2005010078

24. Joh K, Aizawa S, Matsuyama N, et al. Morphologic variations of dense deposit disease: light and electron microscopic, immunohistochemical and clinical findings in 10 patients. Acta Pathol Jpn. 1993;43(10):552-565.

25. Leys A, Vanrenterghem Y, Van Damme B, Snyers B, Pirson Y, Leys M. Sequential observation of fundus changes in patients with long standing membranoproliferative glomerulonephritis type II (MPGN type II). Eur J Ophthalmol. 1991;1(1):17-22. doi:10.1177/ 112067219100100104

26. Dalvin LA, Fervenza FC, Sethi S, Pulido JS. Shedding light on fundus drusen associated with membranoproliferative glomerulonephritis: breaking stereotypes of types I, II, and III. Retin Cases Brief Rep. 2016;10(1):72-78. doi:10.1097/ICB.0000000000000164

27. Khan KN, Mahroo OA, Khan RS, et al. Differentiating drusen: drusen and drusen-like appearances associated with ageing, age-related macular degeneration, inherited eye disease and other pathological processes. Prog Retin Eye Res. 2016;53:70-106.

28. Meyerle CB, Smith RT, Barbazetto IA, Yannuzzi LA. Autofluorescence of basal laminar drusen. Retina. 2007;27 (8):1101-1106. doi:10.1097/IAE.0b013e3181451617

29. Park SP, Siringo FS, Pensec N, et al. Comparison of fundus autofluorescence between fundus camera and confocal scanning laser ophthalmoscope-based systems. Ophthalmic Surg Lasers Imaging Retina. 2013;44(6):536-543. doi:10.3928/23258160-20131105-04

30. Querques G, Guigui B, Leveziel N, et al. Insights into pathology of cuticular drusen from integrated confocal scanning laser ophthalmoscopy imaging and corresponding spectral domain optical coherence tomography. Graefes Arch Clin Exp Ophthalmol. 2011;249 (11):1617-1625. doi:10.1007/s00417-011-1702-0

31. Leng T, Rosenfeld PJ, Gregori G, Puliafito CA, Punjabi OS. Spectral domain optical coherence tomography characteristics of cuticular drusen. Retina. 2009;29(7):988-993. doi:10.1097/IAE.0b013e318 $1 \mathrm{ae} 7113$

32. Shin DH, Kong M, Han G, Han JC, Ham DI. Clinical manifestations of cuticular drusen in Korean patients. Sci Rep. 2020;10(1):11469. doi:10.1038/s41598-020-68493-2

33. Sakurada Y, Tanaka K, Miki A, et al. Clinical characteristics of cuticular drusen in the Japanese population. Jpn J Ophthalmol. 2019;63(6):448-456. doi:10.1007/s10384-019-00692-5

34. Gass JD, Bressler SB, Akduman L, Olk J, Caskey PJ, Zimmerman LE. Bilateral idiopathic multifocal retinal pigment epithelium detachments in otherwise healthy middle-aged adults: a clinicopathologic study. Retina. 2005;25(3):304-310. doi:10.1097/ 00006982-200504000-00009

35. Nagesha CK, Megbelayin EO. Bilateral multifocal retinal pigment epithelium detachment and pachychoroidopathy. Indian J Ophthalmol. 2018;66(4):570-571. doi:10.4103/ijo.IJO_1070_17
36. Sakurada Y, Parikh R, Yannuzzi LA. Cuticular drusen presenting with subretinal drusenoid deposits (Pseudodrusen). Ophthalmol Retina. 2018;2(8):815. doi:10.1016/j.oret.2018.03.009

37. Pfau M, Lindner M, Gliem M, et al. Mesopic and dark-adapted two-color fundus-controlled perimetry in patients with cuticular, reticular, and soft drusen. Eye. 2018;32(12):1819-1830. doi:10.1038/ s41433-018-0183-3

38. Finger RP, Charbel Issa P, Kellner U, et al. Spectral domain optical coherence tomography in adult-onset vitelliform macular dystrophy with cuticular drusen. Retina. 2010;30(9):1455-1464. doi:10.1097/ IAE.0b013e3181e09829

39. Pilli S, Zawadzki RJ, Werner JS, Park SS. High-resolution Fourier-domain optical coherence tomography findings in vitelliform detachment associated with basal laminar drusen. Retina. 2011;31 (4):812-814. doi:10.1097/IAE.0b013e318207d112

40. Cohen SY, Meunier I, Soubrane G, Glacet-Bernard A, Coscas GJ. Visual function and course of basal laminar drusen combined with vitelliform macular detachment. $\mathrm{Br} \quad J$ Ophthalmol. 1994;78 (6):437-440. doi:10.1136/bjo.78.6.437

41. Mrejen-Uretsky S, Ayrault S, Nghiem-Buffet S, Quentel G, Cohen SY. Choroidal thickening in patients with cuticular drusen combined with vitelliform macular detachment. Retina. 2016;36 (6):1111-1118. doi:10.1097/IAE.0000000000000831

42. Coscas F, Puche N, Coscas G, et al. Comparison of macular choroidal thickness in adult onset foveomacular vitelliform dystrophy and age-related macular degeneration. Invest Ophthalmol Vis Sci. 2014;55(1):64-69. doi:10.1167/iovs.13-12931

43. Grenga PL, Fragiotta S, Cutini A, Meduri A, Vingolo EM. Enhanced depth imaging optical coherence tomography in adult-onset foveomacular vitelliform dystrophy. Eur $J$ Ophthalmol. 2016;26 (2):145-151. doi:10.5301/ejo.5000687

44. Sigford DK, Schaal S. Bilateral choroidal neovascularization associated with basal laminar drusen in a 31-year-old. Can J Ophthalmol. 2014;49(3):e80-e82. doi:10.1016/j.jcjo.2014.03.015

45. Sato A, Senda N, Fukui E, Ohta K. Retinal angiomatous proliferation in an eye with cuticular drusen. Case Rep Ophthalmol. 2015;6 (1):127-131. doi:10.1159/000381616

46. Fragiotta S, Kaden TR, Freund KB. Cuticular drusen associated with aneurysmal type 1 neovascularization (polypoidal choroidal vasculopathy). Int J Retina Vitr. 2018;4(1):44. doi:10.1186/s40942-0180148-5

47. Ahmed D, Stattin M, Haas AM, Graf A, Krepler K, AnsariShahrezaei S. Drusen characteristics of type 2 macular neovascularization in age-related macular degeneration. BMC Ophthalmol. 2020;20(1):381. doi:10.1186/s12886-020-01651-2

48. Bressler NM, Munoz B, Maguire MG, et al. Five-year incidence and disappearance of drusen and retinal pigment epithelial abnormalities. Waterman study. Arch Ophthalmol. 1995;113(3):301-308. doi:10.1001/archopht.1995.01100030055022

49. Gass JD. Drusen and disciform macular detachment and degeneration. Arch Ophthalmol. 1973;90(3):206-217. doi:10.1001/ archopht.1973.01000050208006 


\section{Publish your work in this journal}

Clinical Ophthalmology is an international, peer-reviewed journal covering all subspecialties within ophthalmology. Key topics include: Optometry; Visual science; Pharmacology and drug therapy in eye diseases; Basic Sciences; Primary and Secondary eye care; Patient Safety and Quality of Care Improvements. This journal is indexed on PubMed
Central and CAS, and is the official journal of The Society of Clinical Ophthalmology (SCO). The manuscript management system is completely online and includes a very quick and fair peer-review system, which is all easy to use. Visit http://www.dovepress.com/ testimonials.php to read real quotes from published authors.

Submit your manuscript here: https://www.dovepress.com/clinical-ophthalmology-journal 